\title{
JUICIO MORAL Y VALORES INTERPERSONALES EN ESTUDIANTES DE SECUNDARIA DE LAS CIUDADES LIMA Y JAUJA
}

\author{
María luisa Matalinares C. 1 , Lidia Sotelo L., Noemi Sotelo L., Carlos Arenas I., Gloria Diaz A., \\ Alejandro Dioses Ch., Juan Yaringaño L., Raúl Muratta E., Cecllia Pareja F. \\ Universidad Nacional Mayor de San Marcos, Lima, Perú \\ (RECIBIDO EL 25/8/2009, ACEPTADO El 23/11/ 2009)
}

\begin{abstract}
RESUMEN
La investigación realizada tuvo como objetivo establecer si existía o no relación entre el juicio moral y los valores interpersonales de los estudiantes de 4to y 5to de secundaria de Lima y Jauja. Para la realización del proyecto se evaluó a 355 estudiantes de 4to y 5to de secundaria, de ambos sexos, cuyas edades fluctuaron entre los 14 y 18 años de edad, procedentes de diversos centros educativos estatales de Lima y Jauja a quienes se aplicó el test de reflexión socio moral propuesta por Kohlberg y adaptado por Majluf (1986) y el Cuestionario de Valores Interpersonales SIV propuesta por Leonard Gordon adaptado por Higueras (1972) y Pérez (1987). Al ser procesados los resultados se encontró que las variables Juicio Moral y Valores Interpersonales se encuentran asociadas. El juicio moral se relaciona con las dimensiones conformidad, reconocimiento y benevolencia. Por otro lado, se encontró que existen diferencias significativas entre los alumnos de Jauja y Lima en valores interpersonales, pero no ocurre lo mismo con el juicio moral, tanto en Jauja como en Lima los estudiantes responden en forma similar. En las dimensiones de conformidad, reconocimiento y benevolencia de los estudiantes se muestra diferente en función del sexo. Las mujeres obtienen mejores resultados que los varones, no sucede lo mismo en el caso del juicio moral. Por último, se encontraron diferencias significativas entre el juicio moral y los valores en función del grado de instrucción.
\end{abstract}

Palabras claves: Estudiantes, juicio moral, valores interpersonales.

\section{ABSTRACT \\ MORAL JUDGMENT AND INTERPERSONAL VALUES IN STUDENTS OF HIGH SCHOOL OF CITIES FROM LIMA AND JAUJA}

This research had like a purpose to establish a relation between the Moral Judgment and the Interpersonal Values of the students of 4th. and 5th. of High School, from male and female, whose ages were of 14 to 18 years old, coming from various state's educative institutions, from Lima and Jauja, they answered a Development of Sociomoral Reflection Test by Kohlberg, adapted by Majluf (1986) and the Survey of Interpersonal Values (SIV) by Leonard Gordon, adapted by Higueras (1972) and Perez (1987). The results were processed by the squared Chi, and it was founded that Moral Judgment and Interpersonal Values are

1 Docente Principal de la Facultad de Psicología de la UNMSM. E-mail: maria_luisa93@hotmail.com 
correlated. The Moral Judgment is related to the dimensions of Conformity, Recognition and Benevolence. Besides, data were processed with the U of Mann-Whitney Test showing highly significant differences between the students of Jauja and Lima at interpersonal values test; however, with the moral judgment it does not happen the same, since the students from Jauja and Lima they respond of similar form. In dimensions of Conformity, Recognition and Benevolence, there were differences in relation to the sex, being women who obtained better results than men, not happened the same in the case of the Moral Judgment. Finally, there were significant differences between Moral Judgment and Interpersonal Values based on the instruction level.

Keywords: Students, moral judgment, interpersonal values.

\section{INTRODUCCIÓN}

La preocupación por la moral se manifestó desde la antigüedad con Aristóteles primero (Durkheim, 1966) y Platón después; por su parte, San Agustín señalaba que el hombre tiene conciencia moral (Marías, 1974). En la actualidad el planteamiento gira en torno a que los hechos morales consisten en reglas de conducta sancionadas, donde la sanción es consecuencia del acto. Se considera además la existencia de una moral doméstica, una moral profesional, cívica, entre otras. La moral es siempre la obra de un grupo y no puede funcionar más que si este grupo la protege con su autoridad, ejerciendo un poder colectivo (Durkheim, 1982).

Existen varios enfoques en la psicología moral: el enfoque psicoanalítico, el enfoque de aprendizaje social y el enfoque cognitivo evolutivo. En este último enfoque los principales representantes son Jean Piaget y Laurence Kohlberg. Kohlberg profundiza los trabajos de Piaget y se opone a la teoría del aprendizaje social de Bandura respecto a que la conducta moral sea fruto del hábito o del aprendizaje, planteando que uno no puede seguir principios morales si no los comprende o no cree en ellos. Utiliza el término "concepto moral" como modo de evaluación de lo bueno y de lo recto (Hersh, Reimer y Paolito, 1984). Kohlberg establece dos factores importantes para el desarrollo cognitivo y la interacción entre iguales y/o con los padres; así como las influencias ambientales y/o culturales. Habla de una consecuencia evolutiva en término de etapas considerando la invariabilidad necesaria en la sucesión de etapas. Señala que las etapas son las mismas en los diversos países al margen del contexto cultural.

Kohlberg plantea tres niveles del juicio moral y cada uno de ellos se subdivide en dos etapas o estadios. Los primeros seis estadios se caracterizan por ser invariantes en el orden que alcanzan, ser universales, formar estructuras en conjunto y estar jerárquicamente interrelacionados. Cabe anotar que cada estadio es cualitativamente distinto al anterior y está relacionado con la edad dentro de un agrupamiento general.

El primer nivel se denomina moral preconvencional, este nivel se divide en dos etapas: La primera etapa está relacionada al castigo y la obediencia (heterenomía). El punto de vista propio de esta etapa es el egocéntrico, no se reconocen los intereses de los otros como diferentes a los propios. Las acciones se consideran sólo físicamente, no se consideran las intenciones, y se confunde la perspectiva de la autoridad con la propia. 
La segunda etapa del primer nivel se centra en el propósito y el intercambio (individualismo). La perspectiva característica de esta etapa es el individualismo concreto. Se desligan los intereses de la autoridad y los propios, y se reconoce que todos los individuos tienen intereses que pueden no coincidir. De esto se deduce que lo justo es relativo, ya que está ligado a los intereses personales, y que es necesario un intercambio con los otros para conseguir que los propios intereses se satisfagan.

El segundo nivel se denomina moral convencional, este nivel se divide en dos etapas:

La primera etapa alude a las expectativas, relaciones y conformidad interpersonal (mutualidad). La perspectiva de esta etapa consiste en ponerse en el lugar del otro: es el punto de vista del individuo en relación con otros individuos. Se destacan los sentimientos, acuerdos y expectativas compartidas, pero no se llega aún a una generalización del sistema.

La segunda etapa enfatiza sobre el sistema social y conciencia (ley y orden). En este nivel el punto de vista desde el cual el individuo ejerce su moral se identifica en esta etapa con el del sistema social que define los papeles individuales y las reglas de comportamiento. Las relaciones individuales se consideran en función de su lugar en el sistema social y se es capaz de diferenciar los acuerdos y motivos interpersonales del punto de vista de la sociedad o del grupo social que se toma como referencia.

El tercer nivel denominado moral posconvencional o basada en principios. Las decisiones morales en este nivel tienen su origen en el conjunto de principios, derechos y valores que pueden ser admitidos por todas las personas que componen la sociedad, entendiéndose ésta como una asociación destinada a organizarse de un modo justo y beneficioso para todos sin excepción. A decir de Kolhberg, en esta última etapa se alcanza por fin una perspectiva propiamente moral de la que se derivan los acuerdos sociales. Es el punto de vista de la racionalidad, según el cual todo individuo racional reconocerá el imperativo categórico de tratar a las personas como lo que son, fines en sí mismas, y no como medios para conseguir ninguna ventaja individual o social.

A nivel internacional se han desarrollado diversas investigaciones respecto del juicio moral.

Chamblas, Mathiesen, Mora y Navarro (2004) realizaron una investigación en Concepción, Chile, con 546 jóvenes estudiantes de enseñanza media, cuyas edades fluctuaban entre los 12 y 18 años, a fin de conocer el efecto de las variables funcionamiento familiar sobre la permisividad y el juicio moral. Evaluaron la muestra con la Escala de comportamientos morales controvertidos de Harding y Phillips que mide el grado de permisividad respecto de la sexualidad, legalidad y honorabilidad personal y la Prueba de reflexión sociomoral (SROM-SF) en su forma corta de Gibbs y Basinger, el Apgar Familiar de Feetan que mide la insatisfacción con el funcionamiento familar, y por último la Escala de familismo de Bardis. Los resultados mostraron, entre otras cosas, que los estudiantes son menos permisivos en moralidad personal y más permisivos en el ámbito sexual. Los hijos de padres o madres universitarios mostraron mayor proporción de juicio moral bueno o muy bueno, que los hijos de padres con otros niveles educacionales.

Retuerto (2004) investigó en Valencia el desarrollo del razonamiento moral, razonamiento moral prosocial y empatía en la adolescencia y juventud. Trabajó con una muestra de 
556 sujetos de ambos sexos, de edades comprendidas entre los 13 y los 23 años, y cuyos niveles educativos son $3^{\circ} \mathrm{ESO}, 2^{\circ} \mathrm{BUP}, 3^{\circ} \mathrm{BUP}, \mathrm{COU}, 1 \mathrm{er}$ ciclo universitario y $2^{\circ}$ ciclo universitario. Los resultados mostraron que el sexo no produce ningún efecto significativo sobre el razonamiento moral de los adolescentes y jóvenes. Chicos y chicas obtienen similares puntuaciones. En razonamiento prosocial, el sexo origina diferencias significativas. Los varones puntúan más que las mujeres en razonamiento orientado a la aprobación (aunque sólo los adolescentes), mientras que las mujeres puntúan más que los varones en los razonamientos estereotipado, internalizado y puntuación compuesta PROM. En empatía también aparecen diferencias significativas debidas al sexo, de forma que varones y mujeres tienen una capacidad cognitiva similar para comprender la situación del otro, pero las mujeres están más predispuestas a una respuesta afectiva ante dicha situación. La edad ejerce una influencia significativa sobre el razonamiento moral, sobre el razonamiento prosocial y sobre la empatía de los adolescentes y jóvenes. Respecto al razonamiento moral, se produce con la edad una transición gradual de una moral convencional a una moral derivada de un código racional, de una moral sociónoma a una moral autónoma. En razonamiento prosocial, los juicios morales llegan a ser menos egocéntricos y más orientados al otro, así como más abstractos, con el avance de la edad. En empatía, a medida que incrementa la edad de los adolescentes y jóvenes aumentan progresivamente las puntuaciones en toma de perspectiva, fantasía y preocupación empática. El nivel educativo tiene un efecto significativo sobre el razonamiento moral, sobre el razonamiento prosocial y sobre la empatía de los adolescentes y jóvenes. Tal efecto es similar al producido por la edad. El razonamiento moral, el razonamiento prosocial y la empatía están interrelacionados empíricamente de forma positiva. Dado que en cada uno de estos tres constructos están implicados procesos cognitivos y afectivos, y que ellos están interrelacionados empíricamente de forma positiva, se concluye, a su vez, una relación positiva entre los procesos cognitivos y afectivos en el desarrollo moral de la persona.

Barba y Romo (2005) presentan la evaluación del desarrollo moral de estudiantes de educación superior con base en la teoría de L. Kohlberg. Se analiza su perfil moral según las variables semestre, género, institución, carrera, edad y nivel educativo. Los principales resultados son: el nivel de moralidad predominante es el convencional; las mujeres de una institución privada aventajan a los hombres en la moralidad de principios, entre otros.

García y Pérez (2005) realizaron una investigación con 323 estudiantes universitarios. Los resultados obtenidos utilizando el Defining Issues Test-DIT de J. Rest y la Escala de valores (A) de Milton Rokeach, indican que a la convencionalidad moral se asocia una constelación de valores diferente a la que se asocia a la posconvencionalidad moral, en términos estadísticamente significativos. Todo ello permite concluir que el avance del razonamiento moral a través de los estadios de desarrollo moral (estructura del razonamiento moral) se ve acompañado de cambios en la jerarquía de valores asociada (contenido del razonamiento moral), que estructura y contenido del razonamiento moral no son independientes.

Echevarría y Vasco (2006) estudiaron las justificaciones morales sobre lo bueno y lo malo de niñas y niños de contextos considerados violentos y no violentos de una ciudad de la zona andina colombiana. Se utilizaron talleres pedagógicos durante los cuales los niños y niñas manifestaron y ejemplificaron de diversas maneras (dibujos, dramatizaciones, discusiones de grupo, entrevistas grupales) lo que para ellos era lo bueno y lo malo y sus 
razones para ello. Se utilizó un diseño de estudio de caso múltiple de dos casos y en cada contexto se trabajó con tres niños y dos niñas que cursaban cuarto grado y tenían entre los 9 y los 11 años. Todos los talleres e interacciones con los niños y niñas se grabaron en audio y se transcribieron. Para su análisis se utilizó la técnica de análisis del discurso propuesta por Van Dijk (2000). Los resultados mostraron que los niños y niñas de ambos contextos comparten algunas preocupaciones morales tales como la preservación de la naturaleza, la protección de la vida y el mantenimiento del orden vigente. También se encontraron diferencias. Entre ellas, en el contexto violento los niños y las niñas enfatizaron la preservación de la propia vida y la lealtad. En el contexto no violento se encontró un marcado énfasis en la perspectiva política con referencia al orden legalmente establecido. Se concluyó que el énfasis de los niños y niñas del contexto violento en la lealtad como principio de acción requiere mayor estudio porque podría configurar un posible tercer principio moral de regulación más allá de los dos principios ya establecidos de la justicia y del cuidado.

Ortiz, Apodaca, Etxebarria, Fuentes y López (2008) analizaron la relación entre el afecto, la aceptación, la comunicación emocional, la transmisión de valores y la intervención moral de los padres, y las emociones y conductas morales (empatía, culpa, conducta internalizada y reparación) de los hijos; y conocer qué variables familiares explican la internalización moral en niños y niñas. La muestra estuvo formada por los padres y madres de 485 sujetos (244 niños y 241 niñas) de 6 a 8 años. Se encontró que la principal variable predictora de la capacidad de control de la conducta en las niñas fue el afecto materno, mientras que en los niños lo fueron la comunicación emocional y la transmisión de valores maternos. Las variables familiares que explican la internalización moral en los grupos extremos de niños y niñas fueron fundamentalmente el afecto, la comunicación emocional, la transmisión de valores y la intervención moral de padres y madres.

Romo (2005) en México investigó el desarrollo del juicio moral y la solución de la crisis de identidad en estudiantes del bachillerato de Aguascalientes. Los principales resultados mostraron que el nivel de razonamiento moral predominante fue el convencional; en moralidad posconvencional se obtuvieron puntuaciones por debajo de otros estudios; la única variable relacionada con el juicio moral fue el género, en favor de las mujeres.

Ahora bien, en el Perú, Grimaldo (2002) realizó la adaptación del Cuestionario de reflexión socio moral (SROM) de Gibbs y Widaman en estudiantes 260 de tercero, cuarto y quinto de secundaria de un colegio estatal y estudiantes de 1er y 3er año de la Facultad de Psicología de una universidad estatal. Concluyendo que el Cuestionario de reflexión socio moral (SROM) constituye un instrumento válido y confiable para medir el desarrollo del juicio moral, además observó una progresión del juicio moral en la medida que aumenta la edad, además no encontró diferencias respecto de la variable sexo.

Altez (2004) presenta el desarrollo del juicio moral en 545 estudiantes universitarias, según edad, ciclo y programa académico. Los resultados mostraron que no existen diferencias significativas entre las edades y ciclos académicos, pero sí existe diferencia entre programas académicos. La muestra se ubica en el estadio 4 en primer lugar, el estadio 3 en segundo lugar y el estadio 5 en el tercer lugar. Se presenta un baremo basado en la muestra investigada. 
Meneses (2005) realizó un estudio con adolescentes de 4to y 5to de secundaria que estudiaban en un colegio militarizado con régimen de internado y otro grupo de adolescentes que no estudiaban en Colegio militarizado, ambos grupos fueron evaluados en juicio moral con el Cuestionario de Di BLassio, Pagnani, Pedrabassi y Venuni encontrando que no existen diferencias en el juicio moral entre los adolescentes de ambos grupos.

Respecto al valor, cabe señalar que es una cualidad por la que una persona, un hecho, despierta mayor o menor aprecio, admiración o estima (Conalep, 1998). Los valores concuerdan con nuestras concepciones de la vida y del hombre; no existen en abstracto ni de manera absoluta, se encuentran ligados a la historia, las culturas, los grupos humanos; influyen en nuestra forma de pensar, en nuestros sentimientos y formas de comportarnos; suponen un compromiso real y profundo de la persona ante sí misma y ante el medio social donde vive. Existen dos grandes grupos de valores que se dan en la sociedad: los valores relacionados con el desarrollo de una moral ciudadana y los valores que permiten a los individuos construir sus patrones autonomía, ser solidarios y vivir comprometidos con su sociedad. La escuela es la institución educativa importante que proporciona los cimientos que preparan al ser humano para la vida, impartiendo no sólo conocimientos sino desarrollando y profundizando las actitudes y valores que requiere el estudiante a nivel intelectual, interpersonal y moral. Según Gordon (1977), los valores pueden constituir un medio para determinar lo que los sujetos hacen y cómo lo hacen; muchas de sus decisiones inmediatas o sus planes a largo plazo están influidos consciente o inconscientemente por el sistema de valores que adopten. Tal autor desde un enfoque factorial ha podido identificar seis factores o valores interpersonales, valores cuya fuerza en cada sujeto puede determinarse mediante su estudio y son: Soporte, Reconocimiento, Conformidad, Independencia, Benevolencia y Liderazgo (Gordon, 1991). En el extranjero investigadores como Alonso, Pereira y Soto (2003); Alcalde, Marchena y García (2006) han realizado una serie de estudios sobre los valores.

En el Perú se han realizado algunas investigaciones con los valores interpersonales. Rodríguez y Capote (2002) realizaron un análisis del bienestar subjetivo: Valores y moldes cognitivos en un grupo de adolescentes. Encontraron que predominan los valores del nivel primario con mayor facilidad de conseguir, pero al mismo tiempo, más efímeros. En cuanto a los moldes cognitivos, señalaron la supremacía de moldes cognitivos que llevan a actuar sin mucha implicación en la tarea y de manera impulsiva, con cierta disociación emocional, viendo las cosas de manera fría y distante. En cuanto al bienestar subjetivo, el grupo muestral tiene mayor bienestar general que el de la población. En este sentido, señalaron la importancia y el valor que le dan al disfrute con los otros y, a las relaciones interpersonales y afectivas como aquello que les genera mayor bienestar. Se observa la mayor disposición de los hombres a valorar el disfrute social, a valorar lo lúdico y festivo, lo estético y lo intelectivo. Los hombres manifiestan un bienestar subjetivo mayor que las chicas atribuyendo a las relaciones afectivas e interpersonales como variables que más influyen en ese bienestar. Las mujeres dan un valor significativo a la norma y a la higiene; usan moldes más relacionados con la pasividad y la reflexión, al mismo tiempo que manifiestan menor bienestar subjetivo individual que los hombres. Otro aspecto a señalar es la mayor persistencia y estabilidad temporal del bienestar subjetivo en los hombres que en las mujeres. 
Cornejo y Dávila (2002) hallaron en alumnos de una institución militar que mientras un 47,4\% se encuentran en el estadio 2 (Propósito e intercambio instrumentales) un $50.3 \%$ se encuentran en el estadio 3 (Mantenimiento de relaciones mutuas, aprobación de los demás) que coincide al nivel II de moral convencional. Estos resultados se compararon con una muestra de estudiantes de una institución educativa no militar encontrándose que no se presentan diferencias significativas con los estudiantes de instituciones militares.

Aguilar (2002) realizó un estudio comparativo de los valores interpersonales en alumnos con alta y baja autoestima con estudiantes universitarios de los tres primeros ciclos de la escuela de Psicología de la Universidad Señor de Sipán de Chiclayo. Los resultados mostraron que existen diferencias significativas en el estudio del nivel soporte como valor interpersonal entre los estudiantes con alta y baja autoestima. Existen diferencias significativas en el estudio del valor conformidad. No existen diferencias significativas en el valor reconocimiento. Existen diferencias significativas en el valor independencia. No existen diferencias significativas en el valor interpersonal de benevolencia. Existen diferencias significativas en el valor liderazgo. Los alumnos de alta autoestima poseen puntajes altos en los valores: liderazgo y benevolencia. Los alumnos con baja autoestima tienden a presentar bajo nivel en los valores de: soporte, conformidad, reconocimiento e independencia.

Pareja (2004) investigó la inteligencia emocional y su relación con los valores interpersonales en estudiantes de quinto de secundaria. En una muestra de 376 estudiantes de ambos sexos, procedentes de niveles socioeconómicos medio y bajo de Lima Metropolitana, a quienes evaluó con el Ice de Bar-On. Los resultados mostraron que existía una correlación positiva entre la inteligencia emocional y los valores interpersonales de benevolencia y conformidad, lo que implica que los estudiantes de 5to. de secundaria presentan un mayor desarrollo de la inteligencia emocional, caracterizada por una capacidad adecuada y saludable, vinculada con la ayuda al prójimo y una disposición para hacer lo que socialmente es correcto. No halló diferencias significativas respecto de la variable sexo.

Guerrero (2005) estudió la relación entre los acontecimientos vitales críticos y los valores interpersonales en los alumnos del 5to. de secundaria. Encontró que existe relación entre los valores interpersonales de reconocimiento y los sucesos críticos vitales. Los resultados mostraron que existe una relación inversa entre el valor interpersonal independencia y los sucesos críticos vitales. No existe relación significativa entre los valores interpersonales de conformidad y benevolencia y los sucesos críticos vitales.

Murakami (2005) investigó los valores interpersonales en alumnos de 5to año de educación secundaria en colegios estatales mixtos de Lima y Piura. Trabajó con 350 estudiantes de 5to de secundaria de Lima y Chulucanas. La investigadora encontró que los estudiantes de Lima tienen una mayor tendencia a rebelarse hacia las normas impuestas debido a que se encuentran en una constante lucha por alcanzar estabilidad y seguridad en la vida. A su vez, no sienten la necesidad de ser reconocidos por otros posiblemente debido a que ya se sienten o "se saben" reconocidos, pero evitan demostrarlo ya que podrían ser mal vistos o considerados "jactanciosos" o "petulantes" por los demás, así también valoran más la independencia lo cual refleja un accionar más individualista, buscando el beneficio propio, que podría ser causado por la gran competencia que existe en Lima donde cada uno tiene que velar por sí mismo y luchar constantemente por sobresalir. Entre los adolescentes 
de Chulucanas se encontró que se presentan como individuos que tienden a respetar las leyes y normas impuestas debido a que se sienten seguros como están, vale decir, no protestan; esto podría deberse a que sus oportunidades de desarrollo y aspiraciones en la vida son limitadas en donde viven. Además, sienten la necesidad de ser reconocidos por otros -cosa que no ocurre en la capital-, ya sea por sus habilidades o su importancia dentro de la sociedad, lo cual refleja un sentimiento de descontento o inferioridad ante el hecho de percibirse como poco conocidos y un tanto olvidados. Finalmente, tienen poca preferencia por el valor de independencia, lo cual denota una mayor inclinación por hacer las cosas pensando en las necesidades e intereses de su grupo o comunidad debido a que en su entorno, el nivel de competitividad es menor que en Lima, y sumado a sus niveles de conformismo, no se preocupan mayormente por sobresalir, sino más bien, por llevar una vida en comunidad y en familia.

Carmona y León (2005) citados por Romero y Villegas (2006) investigaron la relación entre la inteligencia intrapersonal y los valores interpersonales en estudiantes del primer ciclo de Psicología de la Universidad César Vallejo de Trujillo. Encontraron que la inteligencia intrapersonal y los valores interpersonales son variables independientes que no guardan relación entre ellas. El componente liderazgo correlaciona con la inteligencia intrapersonal. El valor interpersonal de liderazgo correlaciona con la inteligencia interpersonal.

Romero y Villegas (2006) realizaron una comparación de los valores interpersonales de los estudiantes de 1 er. ciclo con los del 11 ciclo. Los resultados mostraron que los alumnos del primer ciclo tienen mayor predominio de la escala de soporte, quienes tienen mayor necesidad de contar con la consideración y aprobación de los demás, en tanto que los alumnos de undécimo ciclo denotan menos necesidad de apoyo y comprensión de los demás. Los alumnos del primer ciclo de Psicología tienden a una mayor necesidad de reconocimiento, en tanto que los del undécimo, revelan menor necesidad de reconocimiento. El valor de independencia y autonomía predominan en el grupo del undécimo ciclo, en tanto que los alumnos del primer ciclo denotan una tendencia hacia una adecuada socialización. La Benevolencia es un valor que se presenta con mayor énfasis en el grupo del primer ciclo, a diferencia de los del undécimo ciclo, quienes parecen ser más objetivos y realistas con las necesidades ajenas. El valor de liderazgo aún no se halla afianzado en alumnos del primer ciclo de la carrera de Psicología; en tanto que, en los alumnos del undécimo ciclo existe un mayor predominio de este valor. A menor experiencia universitaria, existe mayor necesidad de soporte y consideración de los demás. Es decir, la experiencia universitaria, posibilita mayor seguridad en los sujetos del estudio. No se hallan diferencias estadísticamente significativas en el valor de conformidad entre los estudiantes de primer y undécimo ciclo de la carrera profesional de Psicología. El valor de reconocimiento coexiste como una necesidad en los alumnos ingresantes y va desapareciendo esta búsqueda de reconocimiento a medida que se acrecienta la experiencia profesional. La escala de independencia evoluciona favorablemente en la medida que se desarrolla otras habilidades como las académicas, las de formación personal, entre otras que permita actuar con mayor autonomía a medida que estas evolucionan. La benevolencia es un valor que coexiste en los estudiantes de psicología, pero que va evolucionando hacia una mayor objetividad y realismo en la percepción de los problemas humanos, a medida que la formación en psicología se acrecienta. El liderazgo, es un valor en las relaciones interpersonales que 
evoluciona favorablemente a medida que se va consolidando la formación profesional del psicólogo.

\section{HIPÓTESIS}

\section{Hipótesis general}

El juicio moral y los valores interpersonales de los estudiantes que cursan el 5to. grado de secundaria de Lima y Jauja se relacionan significativamente.

\section{Hipótesis específicas}

$\mathbf{H}_{1}$ Existen diferencias significativas en juicio moral de estudiantes que cursan el 5to. grado de secundaria en función del lugar de procedencia.

$\mathbf{H}_{2}$ Existen diferencias significativas en valores interpersonales de estudiantes que cursan el 5to. grado de secundaria en función del lugar de procedencia.

$\mathbf{H}_{3}$ Existen diferencias significativas en juicio moral de estudiantes que cursan el 5to. grado de secundaria en función del sexo.

$\mathbf{H}_{4}$ Existen diferencias significativas en valores interpersonales de estudiantes que cursan el 5to. grado de secundaria en función del sexo.

$\mathbf{H}_{5}$ Existe relación significativa entre los valores interpersonales y juicio moral de estudiantes que cursan el 5to. grado de secundaria en función de la ciudad de procedencia.

$\mathbf{H}_{6}$ Existe relación significativa entre los valores interpersonales y juicio moral de estudiantes que cursan el 5to. grado de secundaria en función del sexo.

\section{MÉTODO}

En concordancia con la hipótesis formulada se empleó en un primer momento el método descriptivo con un diseño correlacional para relacionar las dos variables y luego el diseño descriptivo comparativo para comparar los resultados de la zona rural con la zona urbana.

Sujetos: Se tomó en forma estratificada y por cuotas una muestra de 355 estudiantes de ambos sexos, cuyas edades fluctuaron entre los 14-16 años, que cursaban 5to. grado de secundaria de centros educativos nacionales de Lima y Jauja. Las variables principales del estudio fueron: valores interpersonales y juicio moral.

\section{Técnicas e instrumentos}

Dado el carácter de los fenómenos estudiados se utilizaron la técnica psicométrica y de observación indirecta. 
Los instrumentos utilizados fueron:

1. Test de reflexión socio moral propuesto por Leonard Gordon y Pérez, traducido y adaptado a nuestra realidad por Majluf (1983), citado por Grimaldo (1999). Esta prueba implica el uso de dilemas morales. En esta prueba los examinados deben escoger entre seis alternativas, cinco alternativas válidas, que corresponden a cada una de las etapas de juicio moral y una pseudoalternativa. Las respuestas se tabulan y computan hasta llegar a un puntaje que se traduce en una etapa global de juicio moral, cuyo rango de equivalencia con la escala de niveles de juicio moral de Kohlberg permite ubicar al sujeto en el nivel de madurez que le corresponde. La administración puede ser en forma individual o grupal en un tiempo aproximado de 50 minutos. Se puede tomar a jóvenes desde los 14 años. La confiabilidad del test fue determinada por medio del test retest, excluyendo la edad, arrojando una correlación de 0.82 a 0.76. La consistencia interna estimada por medio del Coeficiente de Crombach fue de 0.84. La validez concurrente de esta prueba con la medida SRM (Medida de reflexión sociomoral) fue de $0.77, \mathrm{p}$ menor 0.01 (Grimaldo, 1999).

2. El cuestionario de valores interpersonales SIV propuesto por Leonard Gordon, fue traducido y adaptado por Leonardo Higueras (1972) y Walter Pérez actualizó los baremos en 1987. Esta Prueba fue elaborada con las técnicas del análisis factorial y como un cuestionario de elección en triadas (30 en total, lo que hace un conjunto de 90 ítems) permite apreciar y comparar la importancia que una persona da a los siguientes valores, actitudes o aspectos de sus relaciones con los demás: soporte, conformidad, reconocimiento, independencia, liderazgo. La administracion puede ser grupal y no hay límite de tiempo, aunque toma aproximadamente 20 minutos. La corrección se realiza a mano, mediante la aplicación de plantillas de corrección directamente sobre las hojas de respuestas. La puntuación varía entre 2, 1 ó 0 puntos. La prueba se puede tomar a jóvenes a partir de los 14 años.

\section{Procedimientos}

La recolección de la información se efectuó de manera colectiva, aplicándose en las respectivas aulas de los sujetos: Test de reflexión socio moral y el cuestionario de valores interpersonales cada uno de los cuales estuvo a cargo de dos examinadores. Los instrumentos se administraron siguiendo las normas establecidas en los respectivos manuales.

Las respuestas fueron calificadas en forma manual y se hizo una base de datos que fue procesada mediante el paquete estadístico SPSS versión 13 en inglés. 


\section{RESULTADOS}

Tabla N. ${ }^{0}$ 1. Resultados de la Prueba de Kolmogorov-Smirnov de bondad de ajuste a la curva normal para los resultados del test de valores interpersonales (S.I.V.) y juicio moral (S.R.O.M.)

\begin{tabular}{lcc}
\hline & \multicolumn{2}{c}{ Subtest del S.I.V. } \\
\cline { 2 - 3 } & \multicolumn{1}{c}{ Índice K-S } & $\mathbf{p}$ \\
\hline Valores interpersonales & & \\
Soporte & 1,847 &, 002 \\
Conformidad & 2,438 &, 001 \\
Reconocimiento & 2,055 &, 001 \\
Independencia & 2,174 &, 001 \\
Benevolencia & 1,700 &, 006 \\
Liderazgo & 1,324 &, 060 \\
Juicio moral & 1,634 &, 010 \\
\hline $\mathrm{p}<0.05$ & & \\
$\mathrm{~N}=355$ & &
\end{tabular}

De acuerdo con la Tabla N. ${ }^{\circ} 1$, el estadístico Kolmogorov-Smirnov para la variable valores interpersonales es en el caso de soporte 1,847 , conformidad 2,438, reconocimiento 2,055 , independencia 2,174 , benevolencia 1,700 y liderazgo 1.324 siendo los niveles críticos menores de 0,05 en los casos de soporte, conformidad, reconocimiento, independencia y benevolencia por lo tanto se rechaza la hipótesis de normalidad y concluimos que las puntuaciones de esta variable no se ajustan a la distribución normal. En el caso de liderazgo y juicio moral, los niveles críticos son mayores a 0,05 , por lo tanto, se acepta la hipótesis de normalidad y concluimos que las puntuaciones de esta variable se podrían ajustar a la distribución normal. En el caso de la variable lenguaje comprensivo su nivel de significación es mayor que 0.05 , concluimos que sus puntuaciones se ajustan a la distribución normal. Por lo tanto, no se pueden utilizar pruebas paramétricas para el análisis estadístico.

Tabla N. ${ }^{\circ}$ 2. Distribución de los alumnos por niveles de juicio moral.

\begin{tabular}{|c|c|c|c|c|c|c|c|c|c|c|c|}
\hline & \multirow{2}{*}{\multicolumn{2}{|c|}{$\begin{array}{c}\text { Estadio 1 } \\
\text { Castigo y } \\
\text { orientación a } \\
\text { la obediencia }\end{array}$}} & \multirow{2}{*}{\multicolumn{2}{|c|}{$\begin{array}{c}\text { Estadio 2 } \\
\text { Orientación } \\
\text { instrumental } \\
\text { relativista }\end{array}$}} & \multirow{2}{*}{\multicolumn{2}{|c|}{$\begin{array}{c}\text { Estadio } 3 \\
\text { Orientación a } \\
\text { la concordia } \\
\text { interpersonal }\end{array}$}} & \multirow{2}{*}{\multicolumn{2}{|c|}{$\begin{array}{c}\text { Estadio } 4 \\
\text { Orientación } \\
\text { a la ley y el } \\
\text { orden }\end{array}$}} & \multirow{2}{*}{\multicolumn{2}{|c|}{$\begin{array}{c}\text { Estadio } 5 \\
\text { Orientación } \\
\text { legalista del } \\
\text { contrato }\end{array}$}} & \multirow{3}{*}{ Total } \\
\hline & & & & & & & & & & & \\
\hline & $\mathrm{F}$ & $\%$ & $\mathrm{~F}$ & $\%$ & $\mathrm{~F}$ & $\%$ & $\mathrm{~F}$ & $\%$ & $\mathrm{~F}$ & $\%$ & \\
\hline Total & 2 & 0,6 & 4 & 1,1 & 115 & 32,4 & 223 & 62,8 & 11 & 3,1 & 355 \\
\hline Lima & 1 & 0,4 & 3 & 1.2 & 77 & 31,4 & 155 & 63,3 & 9 & 3,7 & 245 \\
\hline Jauja & 1 & 0,9 & 1 & 0,9 & 38 & 34,5 & 68 & 61,8 & 2 & 1,8 & 110 \\
\hline
\end{tabular}


De la tabla N. ${ }^{\circ} 2$ se puede observar que los alumnos de ambos grupos presentan una mayor frecuencia en el Estadio 4 - Orientación a la ley y el orden (63.3\% en el caso de Lima y 61.8 en el caso de jauja) el menor porcentaje se presenta en el Estadio 1 - Castigo y orientación a la obediencia $(0,4 \%$ en Lima y $0,9 \%)$.

Tabla N. ${ }^{\circ}$ 3. Cálculo de la Prueba chi cuadrado para establecer relación entre el nivel de valores Interpersonales y nivel de juicio moral.

\begin{tabular}{|c|c|c|c|c|}
\hline & \multicolumn{4}{|c|}{ Juicio moral } \\
\hline & Chi - cuadrado & gl & $p$ & \\
\hline Soporte & 11,567 & 8 & 0,210 & \\
\hline Conformidad & 2,152 & 8 & 0,007 & $*$ \\
\hline Reconocimiento & 9,330 & 8 & 0,045 & $*$ \\
\hline Independencia & 0,650 & 8 & 0,395 & \\
\hline Benevolencia & 6,459 & 8 & 0,016 & $*$ \\
\hline Liderazgo & 4,502 & 8 & 0.342 & \\
\hline
\end{tabular}

En la tabla N. ${ }^{\circ}$ 3, se aprecia que el estadístico chi cuadrado toma un valor de probabilidad (nivel crítico o nivel de significación observado) mayor a 0,05 con 8 grados de libertad en las variables soporte, independencia y liderazgo puesto que esta probabilidad es alta, decidimos aceptar la hipótesis de independencia y las variables no están relacionadas. En el caso de conformidad, reconocimiento y benevolencia la probabilidad es menor a 0,05 por lo cual decidimos rechazar la hipótesis de independencia y concluir que estas variables se relacionan con el juicio moral.

Tabla No 4. Cálculo de la Prueba de Kruskal-Wallis para establecer diferencias significativas entre valores interpersonales y nivel de juicio moral.

\begin{tabular}{lrrrrrrrr}
\hline & \multicolumn{9}{c}{ Nivel de juicio moral } & & \\
\cline { 2 - 6 } Valores & Estadio 1 & Estadio 2 & Estadio 3 & Estadio 4 & Estadio 5 & & \\
\cline { 2 - 6 } & $\begin{array}{r}\text { Rango } \\
\text { medias }\end{array}$ & $\begin{array}{c}\text { Rango } \\
\text { medias }\end{array}$ & $\begin{array}{l}\text { Rango } \\
\text { medias }\end{array}$ & $\begin{array}{l}\text { Rango } \\
\text { medias }\end{array}$ & $\begin{array}{l}\text { Rango } \\
\text { medias }\end{array}$ & \multirow{2}{*}{ Chi2 } & \multirow{2}{*}{ p } \\
\hline Soporte & 128,5 & 113,25 & 167,99 & 185,46 & 163,95 & 4,577 & 0,333 \\
Conformidad & 80,5 & 188,63 & 183,43 & 174,49 & 206,32 & 3,287 & 0,511 \\
Reconocimiento & 354,5 & 203,25 & 161,53 & 180,06 & 267,14 & 17,693 & $0,001 *$ \\
Independencia & 21,5 & 92,38 & 187,27 & 176,62 & 168,64 & 8,578 & 0,073 \\
Benevolencia & 51,5 & 218,13 & 191,41 & 173,81 & 131,18 & 8,308 & 0,081 \\
Liderazgo & 329,5 & 233,38 & 176,5 & 176,91 & 167,95 & 5,711 & 0,222 \\
\hline
\end{tabular}

$N=137$

$* \mathrm{p}<0,05$ 
En la Tabla N. ${ }^{\circ}$, se aprecia que el estadístico Kruskal-Wallis toma un valor de probabilidad (nivel crítico o nivel de significación observado) mayor a 0,05 en todos los valores, a excepción del valor interpersonal reconocimiento. Por lo que se decide aceptar la hipótesis de igualdad y concluir que las variables niveles de soporte, conformidad, independencia, benevolencia y liderazgo, no se diferencian significativamente por el nivel de juicio moral que obtengan; con la excepción del valor interpersonal reconocimiento.

Tabla No 5. Prueba de U de Mann-Withney del cuestionario de valores interpersonales y juicio moral en función de la ciudad de procedencia.

\begin{tabular}{lrrrr}
\hline & \multicolumn{2}{c}{ Rango de medias } & \multirow{2}{*}{$\mathbf{U}$} & \multicolumn{1}{c}{$\boldsymbol{p}$} \\
\cline { 2 - 3 } & \multicolumn{1}{c}{ Lima } & \multicolumn{1}{c}{ Jauja } & & \\
\hline Juicio moral & 180,44 & 172,56 & 12877,000 & $0,043^{*}$ \\
Soporte & 179,833 & 173,918 & 13026,000 & 0,614 \\
Conformidad & 179,494 & 174,673 & 13109,000 & 0,681 \\
Reconocimiento & 174,749 & 185,241 & 12678,500 & $0,037^{*}$ \\
Independencia & 175,682 & 183,164 & 12907,000 & 0,524 \\
Benevolencia & 178,376 & 177,164 & 13383,000 & $0,009 *$ \\
Liderazgo & 174,414 & 185,986 & 12596,500 & 0,324 \\
\hline
\end{tabular}

a. $\mathrm{N}=355$

b. Variable de agrupación: ciudad

Se aprecia de los resultados que el nivel crítico $p$ es menor a 0,05 en el caso de Juicio moral, reconocimiento y benevolencia por lo cual rechazamos la hipótesis nula de homogeneidad; es decir, el puntaje en estas áreas no es el mismo en el Lima y Jauja. En las otras áreas examinadas el puntaje es mayor a 0,05 por lo que se acepta la hipótesis de homogeneidad, es decir, no se presentan diferencias significativas en soporte, conformidad, independencia y liderazgo.

Tabla N. ${ }^{\circ}$ 6. Prueba de U de Mann-Whitney del cuestionario de valores interpersonales y juicio moral en función del sexo.

\begin{tabular}{|c|c|c|c|c|}
\hline & \multicolumn{2}{|c|}{ Rango de medias } & \multirow{2}{*}{$\mathbf{U}$} & \multirow{2}{*}{$p$} \\
\hline & masculino & femenino & & \\
\hline Juicio moral & 182,02 & 174,39 & 15032,000 & $0,040 *$ \\
\hline Soporte & 168,869 & 186,203 & 14174,000 & 0,110 \\
\hline Conformidad & 180,024 & 176,182 & 15368,000 & 0,724 \\
\hline Reconocimiento & 172,955 & 182,532 & 14860,500 & 0,378 \\
\hline Independencia & 189,896 & 167,313 & 13709,500 & $0,038 *$ \\
\hline Benevolencia & 178,869 & 177,219 & 15562,000 & 0,880 \\
\hline Liderazgo & 176,417 & 179,422 & 15442,000 & 0,782 \\
\hline
\end{tabular}

a. $\mathrm{N}=355$

b. Variable de agrupación: sexo 
Se aprecia de los resultados que el nivel crítico $p$ es menor a 0,05 en el caso de juicio moral e independencia, por lo cual rechazamos la hipótesis nula de homogeneidad; es decir, el puntaje en esta área no es el mismo para estudiantes de sexo femenino y masculino. En las otras áreas examinadas el puntaje es mayor a 0,05 por lo que se acepta la hipótesis de homogeneidad, es decir, no se presentan diferencias significativas en soporte, reconocimiento, conformidad y liderazgo.

\section{DISCUSIÓN}

Los resultados expuestos en la tabla $\mathrm{N}^{0} 1$ muestran que los puntajes obtenidos en las pruebas de valores interpersonales y juicio moral no se distribuyen normalmente dentro de la población estudiada. Por lo tanto, no se puede esperar que los puntajes de los instrumentos que las evalúan alcancen en la gráfica una curva normal.

En la Tabla N. ${ }^{\circ} 2$ se muestra que poco más del $94 \%$ de la muestra evaluada se encuentra en el nivel convencional del desarrollo del juicio moral; esto significa que el $32.4 \%$ de los alumnos tiende a ponerse en el lugar del otro, destacándose los sentimientos, acuerdos y expectativas compartidas, pero no llegan aún a una generalización del sistema; mientras que el $62.8 \%$ de los alumnos ejerce su moral identificándose con el punto de vista del sistema social que define los papeles individuales y las reglas de comportamiento. Las relaciones individuales son consideradas por los estudiantes en función del lugar que ocupan en el sistema social y siendo capaces de diferenciar los acuerdos y motivos interpersonales del punto de vista de la sociedad. Es notable que solo el 3.5\% de la muestra alcanza el estadio 5 correspondiente al nivel postconvencional; pero es más notable aún, que ningún estudiante alcance el sexto estadio; el más alto en la propuesta de Kolberg (1992).

Estos resultados se condicen con los de Altez (1997) en el Perú; Cortés (2002) Barba y Romo (2005) en España y Romo (2005) en México. En todos estos casos, las poblaciones estudiadas se ubicaron mayoritariamente en el nivel convencional. Es oportuno destacar que tales resultados parecen ser generalizables tanto a estudiantes del nivel secundario como a los de nivel universitario. Otro detalle no menos interesante es que ya en 1995, Rojas había encontrado que los adolescentes de 17 años se encontraban en la transición de la moral preconvencional a la moral convencional.

En lo concerniente a los valores interpersonales los resultados mostraron que existe relación entre los valores interpersonales conformidad, reconocimiento y benevolencia con el juicio moral (véase Tabla N. ${ }^{\circ} 2$ ). Esto significa que los jóvenes que tienden a acatar las normas comunes de convivencia, el ser considerado como una persona importante y que los demás reconozcan que es así y en ser generoso, filántropo, altruista presentan un mayor nivel en desarrollo de juicio moral.

Por lo que se puede observar en la Tabla N. 3 las variables soporte, conformidad, independencia, benevolencia y liderazgo, no se diferencian significativamente por el nivel de juicio moral que obtengan; con la excepción del valor interpersonal reconocimiento, estos resultados parecen contradecirse con los de Aguilar (2002).

Al comparar los resultados en juicio moral y valores interpersonales de los estudiantes de la zona de Lima y Jauja se encontró que existen diferencias altamente significativas a favor de 
los estudiantes de Lima en nivel de juicio moral, pero se presentan diferencias significativas a favor de los estudiantes de Jauja en reconocimiento y benevolencia (véase Tabla N. ${ }^{\circ} 4$ ). Estos resultados indicarían que los estudiantes de Lima han desarrollado mayores niveles de razonamiento moral. Por otro lado, los estudiantes de Jauja se consideran más importantes, respetados y admirados, se sienten libres para tomar decisiones por sí mismos.

Cuando se realiza la comparación de los resultados en juicio moral y valores interpersonales de acuerdo con el sexo se encontró que existen diferencias altamente significativas a favor de los estudiantes de sexo masculino en el nivel de juicio moral y el valor interpersonal independencia (véase Tabla N. ${ }^{\circ}$ 5). Este resultado es semejante a lo reportado por Mathiesen, Mora, Chamblás y Navarro (2004), quienes encontraron diferencias en el juicio moral entre varones y mujeres aunque refieren que las mujeres presentaron mayores proporciones de juicio moral muy bueno y bueno y un promedio de permisividad más bajo. Estos resultados no concuerdan con los hallazgos de Retuerto (2002), quien encontró que varones y mujeres obtienen similares puntuaciones en razonamiento moral, los de Grimaldo (2002), quien no encontró diferencias de género en el juicio moral y, por último, los de Barba (2002), quien reportó que no existen diferencias significativas en los estadios del desarrollo moral entre varones y mujeres. Respecto del valor interpersonal de independencia, nuestros resultados se condicen con los de Gómez (1985) quien halló que los varones presentaban medias altas en este valor que las mujeres.

\section{CONCLUSIONES}

1. Se ha encontrado relación entre el nivel juicio moral y valores interpersonales en estudiantes de 5to. de secundaria de instituciones educativas públicas de Jauja y Lima, evidenciando que las prácticas de los estudiantes de valores morales e interpersonales están asociados entre sí.

2. Se ha encontrado diferencias significativas en el nivel de juicio moral en función del lugar de residencia. Los estudiantes de Lima obtienen un nivel mayor en juicio moral comparados con los alumnos de Jauja.

3. Se ha encontrado diferencias altamente significativas en valores interpersonales en función del lugar de residencia. Los alumnos de Jauja obtienen mayor puntaje en las dimensiones de reconocimiento y benevolencia.

4. Se ha encontrado diferencias significativas en el nivel de juicio moral considerando la variable sexo. Los varones han obtenido puntuaciones más altas que las mujeres.

5. Los hombres difieren de las mujeres en el valor interpersonal independencia, son los primeros obtienen puntuaciones más altas.

\section{REFERENCIAS BIBLIOGRÁFICAS}

1. Aguilar, S. (2002). Estudio comparativo de los valores interpersonales en los alumnos con alta y baja autoestima de los tres primeros ciclos de la escuela de Psicología de la Universidad Señor de Sipán en la ciudad de Chiclayo. Tesis de Licenciatura en Psicología. 
2. Alcalde, C.; Marchena, E. y García M. (2009). Los valores interpersonales. Análisis de una muestra de alumnos de formación profesional de un centro de recuperación de minusválidos físicos. Universidad de Cádiz. Recuperado el 28 de octubre 2009, de http://campus.usal.es/ inico/investigacion/jornadas/jornada1/posters/p6.html.

3. Alonso, M.; Pereira, M. y Soto, J. (2003). La educación en valores a través de la música. Marco teórico y estrategias de intervención. En Benso Calvo, M. y Pereira Domínguez, M. C. (coords.). El profesorado de enseñanza secundaria. Retos ante el nuevo milenio. Concello de Ourense, Fundación Santa María y Universidad de Vigo. Ourense. Edita Aurea, pp. 135-202.

4. Altez, I. (1997). El juicio moral en estudiantes de una universidad en Lima Metropolitana. Tesis de Magíster.

5. Asmat, C. (2000). Relación entre el desarrollo del juicio moral y desarrollo del pensamiento en los alumnos de educación primaria del C.E.A.C. "Jesús Maestro" Año Moche. Trujillo: Universidad Nacional de Trujillo.

6. Barba, B. (2002). Influencia de la edad y de la escolaridad en el desarrollo del juicio moral. Aguas calientes Revista electrónica de investigación educativa. Vol 4 (2), 2002

7. Barba, B. y Romo, J. (2005). Desarrollo del juicio moral en la educación superior. Revista mexicana de investigación educativa, 24 (10), 67-92.

8. Carmona, B. y León, M. (2005). Análisis correlacional entre las inteligencias múltiples interpersonales e intrapersonales y valores interpersonales, en estudiantes de I ciclo de la carrera de Psicología de la Universidad César Vallejo de la ciudad de Trujillo. Tesis de licenciatura.

9. Coon, W. (2000). Psicología del desarrollo. Prentice Hall México.

10. Conalep (1998). Desarrollo humano y calidad. Modulo I. Valores y actitudes. México: Limusa.

11. Cornejo, J. y Dávila, M. (2002). Reflexión moral en alumnos de la institución Educativa Militar Gran Mariscal Ramón Castilla y la Institución Educativa San Felipe de la Ciudad de Trujillo. Tesis de Licenciatura.

12. Cortes, A. (2002). La contribución de la psicología ecológica al desarrollo moral. Un estudio con adolescentes. Universidad de Murcia: Anales de Psicología, Vol. 18 (1), 111-134.

13. Davalos, L. (1998). Valores interpersonales (según test de Leonard V. Gordon, Ph. D.) de los alumnos de sexto semestre de preparatoria de escuelas maristas. Centro de Evaluación y Diagnóstico Marista. Recuperado el 28 de octubre 2009 de http:// www.educadormarista.com/ARTICULOS/GORDNREP.HTM.

14. Durkeim, E. (1966). Lecciones de sociología. Física de las costumbres y el derecho. Buenos Aires: Schapire.

15. Echevarría, C. y Vasco, E. (2006). Justificaciones morales de lo bueno y lo malo en un grupo de niñas y niños provenientes de contextos violentos y no violentos de una ciudad de la zona andina de Colombia. Acta Colombiana de Psicología, Vol. 9 (1), 51-62. 
16. Finardi, M. (1990). El juicio moral del adolescente. Buenos Aires: Editorial Docencia.

17. Frisancho, S. (2002). Investigación diagnóstica: Experiencias, conocimientos, creencias, actitudes y habilidades para la educación en valores en maestros de escuela primaria del Perú. Lima: Ministerio de Educación.

18. García, J. y Pérez, E. (2005). Razonamiento moral y valores: estudio de sus relaciones en un grupo de universitarios españoles. Revista Latinoamericana de Psicología, Vol. 37 (1), pp. 131-148.

19. Guerrero, M. (2005). Relación entre los acontecimientos vitales críticos y los valores interpersonales en los alumnos del 5to. de secundaria del centro educativo nacional El Indoamericano, de Río Seco. Tesis de licenciatura.

20. Graig, G. (1992). Desarrollo psicológico. México: Prentice Hall Hispanoamericana.

21. Gómez, L (1985). Dimensiones de personalidad y valores interpersonales en adolescentes. Revista Latinoamericana de Psicología. Vol 17 (2), pp. 199-203. Bogotá: Fundación Universitaria Honrad Lorenz,

22. Gordon, L. (1991). Cuestionario de valores interpersonales (S.I.V.). Madrid: TEA.

23. Grimaldo P. (2002). Adaptación del cuestionario de reflexión socio moral de Gibbs \& Widaman. Lima: Universidad de San Martín de Porres.

24. Hersh, R., Reimer, J. y Paolito, D. (1984). El crecimiento moral de Piaget a Kohlberg. Madrid: Nancea.

25. Kolhberg, L. (1992). Psicología del desarrollo moral. Bilbao: Biblioteca de Psicología.

26. Grinder R. (2001). Adolescencia. México: Editorial Limusa de C. V.

27. Heiffos, G. (1987). Nivel de juicio sociomoral e inteligencia en docentes de educación inicial del cono norte, Lima: Universidad Peruana Cayetano Heredia.

28. Marías, J. (1974). Historia de la filosofía. Madrid: Manuales de la Revista Moderna.

29. Mathiesen, M., Mora, O.,Chamblás, I. y Navarro, J. (2004). Familia, permisividad y juicio moral en estudiantes de enseñanza media de la provincia de Concepción. Universidad de Concepción: PSYKHE 2004, Vol. 13 (1), pp. 3 -20.

30. Meneses, M. (2005). Juicio moral en adolescentes de un colegio militarizado y colegios no militarizados. Tesis de Magíster en Psicología UNMSM, Lima.

31. Murakami, M. (2005). Valores interpersonales en alumnos de 5to año de educación secundaria en colegios estatales mixtos de Lima y Piura. Tesis de Licenciado en Psicología. Lima: UNIFE.

32. Ortiz, M., Apodaca, P., Etxebarria, I., Fuentes, M. y López, F. (2008). Predictores familiares de la internalización moral en la infancia. Psicothema 2008. Vol. 20 (4), pp. 712-717. 
33. Pareja, C. (2004). La inteligencia emocional y su relación con los valores interpersonales en estudiantes del 5to. año de educación secundaria. Lima: Tesis Maestría de la UNMSM.

34. Retuerto, A. (2002). Desarrollo del razonamiento moral, razonamiento moral prosocial y empatía en la adolescencia y juventud. http://www.tesisenred.net/TDX-0218104173205.

35. Rodríguez, R. (1986). Los valores interpersonales en los universitarios asturianos. Tesis doctoral, Universidad de Salamanca.

36. Rodríguez, D. y Capote, M. (2002). Análisis del bienestar subjetivo: Valores y moldes cognitivos en un grupo de adolescentes. Evaluación e intervención psicoeducativa: Revista interuniversitaria de Psicología de la Educación, N. ${ }^{\circ}$ 8-9, pp. 63-80. Recuperado el 28 de octubre del 2009 de http://dialnet.unirioja.es/servlet/ articulo? codigo $=918224$

37. Romero, J. y Villegas, C. (2006). Análisis Comparativo de los Valores Interpersonales entre los estudiantes de primer ciclo respecto a los de undécimo ciclo de la carrera profesional de Psicología de la Universidad Privada César Vallejo de la ciudad de Trujillo Tesis de Licenciatura en Psicología.

38. Romo, J. (2005). Desarrollo del juicio moral en adolescentes de aguas calientes. Caleidoscopio. Universidad Autónoma de Aguas Calientes. México, 15 (8) pp. 7-27.

39. Rojas, J. (1995). Juicio moral en adolescentes. Tesis de Licenciatura. Lima: Universidad Nacional Mayor de San Marcos. 